

\title{
PARAMETER ESTIMATION BY IMPLICIT SAMPLING
}

\author{
Matthias MorzFeld, Xuemin Tu, \\ JON WilKENING AND ALEXANDRE J. CHORIN
}

\begin{abstract}
Implicit sampling is a weighted sampling method that is used in data assimilation to sequentially update state estimates of a stochastic model based on noisy and incomplete data. Here we apply implicit sampling to sample the posterior probability density of parameter estimation problems. The posterior probability combines prior information about the parameter with information from a numerical model, e.g., a partial differential equation (PDE), and noisy data. The result of our computations are parameters that lead to simulations that are compatible with the data. We demonstrate the usefulness of our implicit sampling algorithm with an example from subsurface flow. For an efficient implementation, we make use of multiple grids, BFGS optimization coupled to adjoint equations, and Karhunen-Loève expansions for dimensional reduction. Several difficulties of Markov chain Monte Carlo methods, e.g., estimation of burn-in times or correlations among the samples, are avoided because the implicit samples are independent.
\end{abstract}

\section{Introduction}

We wish to compute a set of parameters $\theta$, an $m$-dimensional vector, so that simulations with a numerical model that require these parameters are compatible with data $z$ (a $k$-dimensional vector) we have collected. We assume that some information about the parameter is available before we collect the data and this information is summarized in a prior probability density function (pdf) $p(\theta)$. For example, one may know a priori that some of the parameters are positive. The numerical model, e.g., a partial differential equation (PDE), defines the likelihood $p(z \mid \theta)$, which describes how the parameters are connected with the data. Bayes' rule combines the prior and likelihood to find the posterior density

$$
p(\theta \mid z) \propto p(\theta) p(z \mid \theta) ;
$$

see, e.g., [40]. This posterior pdf defines which parameters of the numerical model are compatible with the data $z$. The goal in parameter estimation is to compute the posterior pdf.

MSC2010: 86-08, 65C05.

Keywords: importance sampling, implicit sampling, Markov chain Monte Carlo. 
If the prior and likelihood are Gaussian, then the posterior is also Gaussian, and it is sufficient to compute the mean and covariance of $\theta \mid z$ (because the mean and covariance define the Gaussian). The posterior mean and covariance are the minimizer and the inverse of the Hessian of the negative logarithm of a Gaussian posterior pdf. In nonlinear and non-Gaussian problems, one can compute the posterior mode, often called the maximum a posteriori (MAP) point, by minimizing the negative logarithm of the posterior, and use the MAP point (instead of the mean) as an approximation of the parameter $\theta$. The inverse of the Hessian of the negative logarithm of the posterior can be used to measure the uncertainty of this approximation. This method is sometimes called linearization about the MAP point (LMAP) or the Laplace approximation $[7 ; 24 ; 34 ; 35]$.

One can also use Markov chain Monte Carlo (MCMC) to solve a parameter estimation problem. In MCMC, one generates a collection of samples from the posterior pdf; see, e.g., $[13 ; 16 ; 29 ; 36]$. The samples form an empirical estimate of the posterior, and statistics, e.g., the mean or mode, can be computed from this empirical estimate by averaging over the samples. Under mild assumptions, the averages one computes from the samples converge to the expected values with respect to the posterior pdf as the number of samples goes to infinity. In practice, a finite number of samples is used and successful MCMC sampling requires that one can test if the chain has converged to the posterior pdf. The convergence can be slow due to correlations among the samples.

An alternative to MCMC is to use importance sampling. The idea is to draw samples from an importance function and to attach a weight to each sample such that the weighted samples form an empirical estimate of the posterior (see, e.g., [8]). The efficiency of importance sampling depends on the importance function which in turn defines the weights. Specifically, if the variance of the weights is large, then the weighted samples are a poor empirical estimate of the posterior and the number of samples required can increase quickly with the dimension of the problem [4; $5 ; 9 ; 39]$. For this reason, importance sampling has not been used for parameter estimation problems in which the dimension is usually large. We investigate if implicit sampling which has been used before in online-filtering/data assimilation $[2 ; 10 ; 11 ; 12 ; 30 ; 31 ; 42]$ can overcome this issue.

We will describe how to apply implicit sampling to parameter estimation problems, and it will become clear that an important step in implicit sampling is to minimize the negative logarithm of the posterior pdf, i.e., to find the MAP point. This optimization step identifies the region where the posterior probability is large, i.e., the region where the high-probability samples are. Starting from the MAP point, implicit sampling generates samples in its vicinity to explore the regions of high posterior probability. The optimization in implicit sampling represents the link between implicit sampling and LMAP. In fact, the optimization methods 
used in LMAP codes can be used for implicit sampling; however, implicit sampling captures non-Gaussian characteristics of the posterior, which are usually missed by LMAP.

We illustrate the efficiency of our implicit sampling algorithm with numerical experiments using a problem from subsurface flow [3; 35]. This problem is a common test problem for MCMC algorithms, and the conditions for the existence of a posterior measure and its continuity are well understood [13]. Earlier work on this problem includes [16], where Metropolis-Hastings MC sampling is used, and [17], which uses optimal maps and is further discussed below.

The remainder of this paper is organized as follows. In Section 2, we explain how to use implicit sampling for parameter estimation and discuss an efficient implementation. Numerical examples are provided in Section 3. Conclusions are offered in Section 4.

\section{Implicit sampling for parameter estimation}

We wish to estimate an $m$-dimensional parameter vector $\theta$ from data which are obtained as follows. One measures a function of the parameters $h(\theta)$, where $h$ is a given $k$-dimensional function; the measurements are noisy so that the data $z$ satisfy the relation

$$
z=h(\theta)+r,
$$

where $r$ is a random variable with a known distribution and the function $h$ maps the parameters onto the data. Often, the function $h$ involves solving a PDE. In a Bayesian approach, one obtains the pdf $p(\theta \mid z)$ of the conditional random variable $\theta \mid z$ by Bayes' rule:

$$
p(\theta \mid z) \propto p(\theta) p(z \mid \theta),
$$

where the likelihood $p(z \mid \theta)$ is given by (1) and the prior $p(\theta)$ is assumed to be known.

The goal is to sample the posterior and use the samples to calculate useful statistics. This can be done with importance sampling as follows [8; 25]. One can represent the posterior by $M$ weighted samples. The samples $\theta_{j}, j=1, \ldots, M$, are obtained from an importance function $\pi(\theta)$ (which is chosen such that it is easy to sample from), and the $j$-th sample is assigned the weight

$$
w_{j} \propto \frac{p\left(\theta_{j}\right) p\left(z \mid \theta_{j}\right)}{\pi\left(\theta_{j}\right)} .
$$

A sample corresponds to a set of possible parameter values, and the weight describes how likely this set is in view of the posterior. The weighted samples $\left\{\theta_{j}, w_{j}\right\}$ form an empirical estimate of $p(\theta \mid z)$ so that, for a smooth function $u$, the sum 


$$
E_{M}(u)=\sum_{j=0}^{M} u\left(\theta_{j}\right) \hat{w}_{j}
$$

where $\hat{w}_{j}=w_{j} / \sum_{j=0}^{M} w_{j}$, converges almost surely to the expected value of $u$ with respect to $p(\theta \mid z)$ as $M \rightarrow \infty$, provided that the support of $\pi$ includes the support of $p(\theta \mid z)[8 ; 25]$.

The importance function must be chosen carefully or else sampling can become inefficient. For example, suppose you choose the prior as the importance function. In this case, the weights are proportional to the likelihood. Thus, one first draws samples from the prior and then determines their posterior probability by comparing them with the data. However, the samples one draws from the prior lie in the region where the prior probability is high and this region may not overlap with the region where the posterior probability is high. Two important scenarios in which this happens are:

(i) The prior and likelihood have (almost) disjoint support; i.e., the prior assigns probability mass in a small region of the (parameter) space in which the likelihood is small and vice versa. See Figure 1a.

(ii) The prior is broad; however, the likelihood is sharply peaked. See Figure 1b.

In either scenario, the samples we draw from the prior typically receive a low posterior probability so that the resulting empirical estimate of the posterior is inaccurate. An accurate empirical estimate requires samples with a high posterior probability, and a large number of prior samples may be required to obtain a few

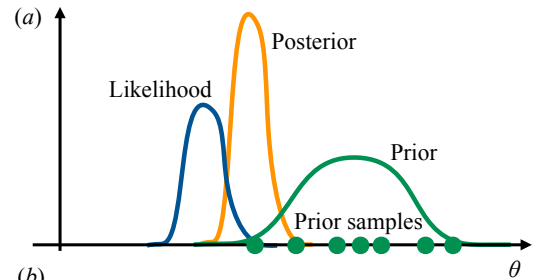

(b)

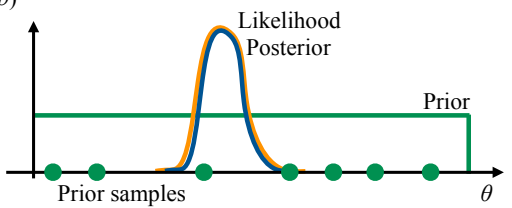

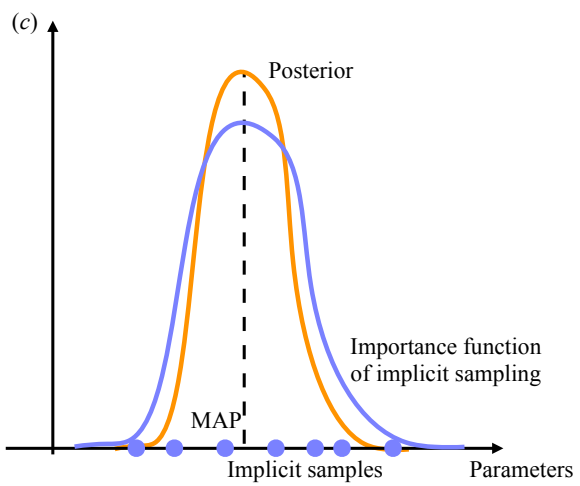

Figure 1. (a) The prior and likelihood are nearly mutually singular so that prior samples receive a small posterior probability. (b) The prior is broad and the likelihood is sharply peaked so that the majority of prior samples receives a small posterior probability. (c) The importance function of implicit sampling assigns probability mass to the neighborhood of the MAP point so that its overlap with the posterior pdf is significant, which leads to implicit samples that receive a large posterior probability. 
samples with high posterior probability. In fact, the number of samples required can increase catastrophically with the dimension of the problem so that this importance sampling algorithm cannot be applied to high-dimensional problems $[4 ; 5 ; 9 ; 39]$.

2.1. Basic ideas of implicit sampling. The idea in implicit sampling is to construct a data-informed importance function, which has a significant overlap with the posterior pdf (see Figure 1c). This requires in particular that the importance function be large where the posterior pdf is large. We can find one point where the posterior pdf is large by minimizing its negative logarithm; i.e., we find the MAP point as in LMAP methods. To set up the notation, let

$$
F(\theta)=-\log (p(\theta) p(z \mid \theta))
$$

so that the MAP point is the minimizer of $F$,

$$
\mu=\underset{\theta}{\arg \min } F(\theta) .
$$

Our goal is to construct an importance function that assigns high probability to the neighborhood of the MAP point. For the construction, we first use a random variable $\xi$ with pdf $p(\xi) \propto \exp (G(\xi))$, which is easy to sample (e.g., a Gaussian). The variable $\xi$ assigns high probability to the neighborhood of its mode, the minimizer of $G$. Next we define a new random variable, $x$, implicitly by the solutions of the algebraic equations

$$
F(x)-\phi=G(\xi)-\gamma,
$$

where $\phi=\min F$ and $\gamma=\min G$. The pdf of $x$ can be calculated by a change of variables

$$
\pi(x)=p(\xi(x))\left|\operatorname{det}\left(\frac{\partial \xi}{\partial x}\right)\right|,
$$

provided the map $\xi \rightarrow x$ is one-to-one and onto. Many one-to-one and onto mappings $\xi \rightarrow x$ exist because (4) is underdetermined: it is a scalar equation in $m$ variables. The pdf $\pi(x)$ is the importance function of implicit sampling, and samples are drawn by solving (4). The weights of the samples are

$$
w_{j} \propto \frac{p(\theta \mid z)}{\pi(x)} \propto \underbrace{\exp (G(\xi(x))-F(\theta))}_{=\exp (\gamma-\phi)=\text { const. }}\left|\operatorname{det}\left(\frac{\partial \xi}{\partial x}\right)\right| \propto\left|\operatorname{det}\left(\frac{\partial \xi}{\partial x}\right)\right|,
$$

proportional to the Jacobian of the map from $x$ to $\xi$.

Note that a typical draw from the variable $\xi$ is close to the mode of $\xi$ so that $G$ evaluated at a typical sample of $\xi$ is close to its minimum $\gamma$. Thus, the left-hand side of (4) is likely to be small. A small left-hand side implies a small right-hand side so that the function $F$ evaluated at the solution of (4) is close to its minimum $\phi$. This forces the solutions of (4) to lie near the MAP point $\mu$. Thus, by repeatedly 
solving (4) for several draws of the variable $\xi$, we explore the neighborhood of the MAP point.

2.2. Solving the implicit equation. We describe and implement two strategies for solving (4) for a Gaussian $\xi$ with mean 0 and covariance matrix $H^{-1}$, where $H$ is the Hessian of the function $F$ at the minimum. With this $\xi$, (4) becomes

$$
F(\theta)-\phi=\frac{1}{2} \xi^{T} H \xi .
$$

Both algorithms are affine invariant and, therefore, capable of sampling within flat and narrow valleys of $F$; see [20] for a discussion of the importance of affine invariance in Monte Carlo sampling.

2.2.1. Random maps. One can look for solutions of (6) in a random direction, $\xi$ :

$$
\theta=\mu+\lambda(\xi) \xi .
$$

The stretch factor $\lambda$ can be computed by substituting (7) into (6) and solving the resulting equation for the scalar $\lambda(\xi)$ with Newton's method. A formula for the Jacobian of the random map defined by (6) and (7) was derived in [22; 31]:

$$
w \propto|J(\xi)|=\left|\lambda^{m-1} \frac{\xi^{T} H \xi}{\nabla_{\theta} F \cdot \xi}\right|,
$$

where $m$ is the number of nonzero eigenvalues of $H$. The Jacobian is easy to evaluate if the gradient of $F$ is easy to compute, e.g., using the adjoint method (see below).

2.2.2. Linear maps. An alternative strategy is to approximate $F$ by its Taylor expansion to second order:

$$
F_{0}(\theta)=\phi+\frac{1}{2}(\theta-\mu)^{T} H(\theta-\mu),
$$

where $\mu=\arg \min F$ is the minimizer of $F$ (the MAP point) and $H$ is the Hessian at the minimum. This strategy is called "implicit sampling with linear maps" and requires that one solves the quadratic equation

$$
F_{0}(\theta)-\phi=\frac{1}{2} \xi^{T} H \xi
$$

instead of (6). This can be done by simply shifting $\xi$ by the mode: $\theta=\mu+\xi$. The bias created by solving the quadratic equation (9) instead of (6) can be removed by the weights $[2 ; 10]$

$$
w \propto \exp \left(F_{0}(\theta)-F(\theta)\right) .
$$

A comparison of the linear and random map methods is given in [22], where it is found that the random map loses its advantages as the dimension of the problem increases if the posterior is a small perturbation of a Gaussian. We will confirm this theory with our numerical examples below. 
2.2.3. Connections with optimal maps. An interesting construction, related to implicit sampling, has been proposed in $[17 ; 38]$. Suppose one wants to generate samples with the pdf $p(\theta \mid z)$ and have $\theta$ be a function of a variable $\xi$ with pdf $g$, as above. If the samples are all to have equal weights, one must have, in the notation above,

$$
p(\theta \mid z)=g(\xi) / J(\xi),
$$

where, as above, $J$ is the Jacobian of a map $\theta \rightarrow \xi$. Taking logs, one finds

$$
F(\theta)+\log \beta=G(\xi)-\log (J(\xi)),
$$

where $\beta=\int p(z \mid \theta) p(\theta) d \theta$ is the proportionality constant that has been elided in (2) and $G(\xi)=-\log \xi$. If one can find a one-to-one mapping from $\xi$ to $\theta$ that satisfies this equation, one obtains an optimal sampling strategy, where the pdf of the samples matches exactly the posterior pdf. In [17], this map is found globally by choosing $g=p(\theta)$ (the prior), rather than sample-by-sample as in implicit sampling. The main differences between the implicit sampling equation (4) and (11) are the presence of the Jacobian $J$ and of the normalizing constant $\beta$ in the latter; $J$ has shifted from being a weight to being a term in the equation that picks the samples, and the optimization that finds the probability mass has shifted to the computation of the map.

If $\xi$ is Gaussian and the problem is linear, (11) can be solved by a linear map with a constant Jacobian and this map also solves (4) so that one recovers implicit sampling. In particular, in a linear Gaussian problem, the local (sample-by-sample) map (4) of implicit sampling also solves the global equation (11), which, for the linear problem, is a change of variables from one Gaussian to another. If the problem is not linear, the task of finding a global map that satisfies (11) is difficult (see also [15; 27; 38; 43]). The determination of optimal maps in [17], based on nonlinear transport theory, is elegant but can be computationally intensive and requires approximations that reintroduce nonuniform weights. Using (simplified) optimal maps and reweighting the samples from approximate maps is discussed in [38]. In [33], further optimal transport maps from prior to posterior are discussed. These maps are exact in linear Gaussian problems; however, in general, they are approximate, due to neglecting the Jacobian, when the problem is nonlinear.

2.3. Adjoint-based optimization with multiple grids. The first step in implicit sampling is to find the MAP point by minimizing $F$ in (3). This can be done numerically by Newton, quasi-Newton, or Gauss-Newton methods (see, e.g., [32]). The minimization requires derivatives of the function $F$.

We consider parameter estimation problems in which the function $h$ in (1) typically involves solving a PDE. In this case, adjoints are efficient for computing the gradient of $F$. The reason is that the complexity of solving the adjoint equation is similar to that of solving the original "forward" model. Thus, the gradient can 
be computed at the cost of (roughly) two forward solutions. Adjoint methods are used widely in LMAP methods and can be used in connection with a quasi-Newton method, e.g., BFGS, or with Gauss-Newton methods. We illustrate how to use the adjoint method for BFGS optimization in the example below.

During the optimization, one can make use of multiple grids. This idea first appeared in the context of online state estimation in [2] and is similar to a multigrid finite difference method [18] and multigrid Monte Carlo [21]. However, the idea is different from the usual "multigrid" method (which is why we call it optimization with multiple grids). The idea is as follows. First, initialize the parameters and pick a coarse grid. Then perform the minimization on the coarse grid and use the minimizer to initialize a minimization on a finer grid. The minimization on the finer grid should require only a few steps, since the initial guess is informed by the computations on the coarser grid, so that the number of fine-grid forward and adjoint solves is small. This procedure can be generalized to use more than two grids (see the example below).

\section{Application to subsurface flow}

We illustrate the applicability of our implicit sampling method by a numerical example from subsurface flow, where we estimate subsurface structures from pressure measurements of flow through a porous medium. This is a common test problem for MCMC and has applications in reservoir simulation/management (see, e.g., [35]) and groundwater pollution modeling (see, e.g., [3]).

We consider Darcy's law

$$
u=-\frac{\kappa}{\mu} \nabla p,
$$

where $\nabla p$ is the pressure gradient across the porous medium, $\mu$ is the viscosity, and $u$ is the average flow velocity; $\kappa$ is the permeability and describes the subsurface structures we are interested in. Assuming, for simplicity, that the viscosity is constant, we obtain, from conservation of mass, the elliptic problem

$$
-\nabla \cdot(\kappa \nabla p)=g,
$$

on a domain $\Omega$, with Dirichlet boundary conditions and where the source term $g$ represents externally prescribed inward or outward flow rates. For example, if a well were drilled and a constant inflow were applied through this well, $g$ would be a delta function with support at the well.

The uncertain quantity in this problem is the permeability; i.e., $\kappa$ is a random variable, whose realizations we assume to be smooth enough so that, for each realization of $\kappa$, a unique solution of (12) exists. We would like to update our knowledge about $\kappa$ on the basis of noisy measurements of the pressure at $k$ locations 


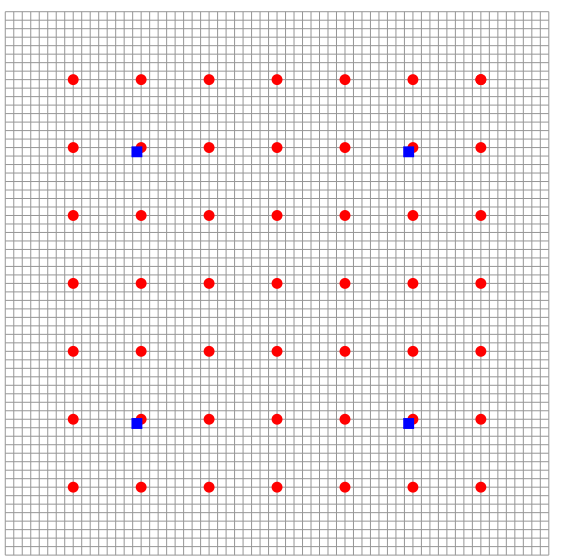

Figure 2. Mesh of the square domain (gray lines), pressure measurements (red dots), and forcing locations (delta distributions, blue squares)

within the domain $\Omega$ so that (1) becomes

$$
z=h(p(\kappa), x, y)+r,
$$

where $r$ is a random variable.

In the numerical experiments below, we consider a $2 \mathrm{D}$ problem on a square domain $\Omega=[0,1] \times[0,1]$ and discretize (12) with a (standard) piecewise linear finite element method on a uniform $(N+1) \times(N+1)$ mesh of triangular elements [6]. We use the balancing domain decomposition by constraints method [14] to solve the resulting symmetric linear systems; i.e., we first decompose the computational domain into smaller subdomains and then solve a subdomain interface problem. The right-hand side $g$ is a superposition of four delta distributions in the center of the domain (see Figure 2).

Our finest grid is $64 \times 64$, and the pressure measurements and forcing $g$ are arranged such that they align with grid points of our fine and coarse grids (which we use in the multiple-grid approach). The 49 pressure measurements are collected in the center of the domain (see Figure 2).

The pressure measurements are perturbed with a Gaussian random variable $r \sim \mathcal{N}(0, R)$, with a diagonal covariance matrix $R$ (i.e., we assume that measurement errors are uncorrelated). The variance at each measurement location is set to $30 \%$ of the reference solution. This relatively large variance brings about significant non-Gaussian features in the posterior pdf.

3.1. The log-normal prior, its discretization, and dimensional reduction. The prior for permeability fields is often assumed to be log-normal, and we follow suit. Specifically, the continuous permeability field is assumed log-normal with a 
squared exponential covariance function [37],

$$
K\left(x_{1}, x_{2}, y_{1}, y_{2}\right)=\exp \left(-\frac{\left(x_{1}-x_{2}\right)^{2}}{l_{x}^{2}}-\frac{\left(y_{1}-y_{2}\right)^{2}}{l_{y}^{2}}\right),
$$

where $\left(x_{1}, y_{1}\right)$ and $\left(x_{2}, y_{2}\right)$ are two points in the domain $\Omega$ and where the correlation lengths are equal: $l_{x}=l_{y}=0.5$. This prior models the (log-)permeability as a smooth function of $x$ and $y$ so that solutions of the PDE (12) uniquely exist. Moreover, the theory presented in $[13 ; 40]$ applies and a well defined posterior also exists for the continuous problem.

The random permeability field is discretized on our uniform grid by a finitedimensional random variable with a log-normal distribution. The elements of the covariance matrix $\Sigma$ are obtained from the continuous correlation function (14)

$$
\Sigma(i, j)=K\left(x_{i}, x_{j}, y_{i}, y_{j}\right), \quad i, j=1, \ldots, N,
$$

where $N$ is the number of grid points in each direction. We perform a dimension reduction via Karhunen-Loève (KL) expansions [19; 26] and use the resulting lowrank approximation of the covariance matrix $\Sigma$ for all subsequent computations. Specifically, the factorization of the covariance function $K\left(x_{1}, x_{2}, y_{1}, y_{2}\right)$ into the $x$ and $y$ directions allows us to compute the covariance matrices in each direction separately; i.e., we compute the matrices $\Sigma_{x}$ and $\Sigma_{y}$ with elements

$$
\Sigma_{x}(i, j)=\sigma_{x}^{2} \exp \left(-\frac{\left(x_{i}-x_{j}\right)^{2}}{l_{x}^{2}}\right), \quad \Sigma_{y}(i, j)=\sigma_{y}^{2} \exp \left(-\frac{\left(y_{i}-y_{j}\right)^{2}}{l_{y}^{2}}\right) .
$$

We then compute singular value decompositions (SVD) in each direction to form lowrank approximations $\hat{\Sigma}_{x} \approx \Sigma_{x}$ and $\hat{\Sigma}_{y} \approx \Sigma_{y}$ by neglecting small eigenvalues. These low-rank approximations define a low-rank approximation of the covariance matrix

$$
\Sigma \approx \hat{\Sigma}_{x} \otimes \hat{\Sigma}_{y}
$$

where $\otimes$ is the Kronecker product. Thus, the eigenvalues and eigenvectors of $\hat{\Sigma}$ are the products of the eigenvalues and eigenvectors of $\hat{\Sigma}_{x}$ and $\hat{\Sigma}_{y}$. We obtain the low-rank approximation for the covariance matrix on the grid from the SVD of the covariance in each direction:

$$
\hat{\Sigma}=V^{T} \Lambda V
$$

where $\Lambda$ is a diagonal matrix whose diagonal elements are the $m$ largest eigenvalues of $\Sigma$ and $V$ is an $m \times N$ matrix whose columns are the corresponding eigenvectors. Our approximate covariance $\hat{\Sigma}$ is optimal in the sense that the difference of the Frobenius norms of $\Sigma$ and $\hat{\Sigma}$ is minimized. With $m=30$ eigenvalues, we capture $99.9 \%$ of the variance (in the sense that the sum of the first 30 eigenvalues is $99 \%$ of the sum of all eigenvalues). 
Thus, in reduced coordinates on the grid, the prior is

$$
\hat{K} \sim \log \mathcal{N}(\hat{\mu}, \hat{\Sigma}) .
$$

Exponentiating followed by the linear change of variables

$$
\theta=V^{T} \Lambda^{-0.5} \hat{K}
$$

gives a prior for the "effective parameters" $\theta$ :

$$
p(\theta)=\mathcal{N}\left(\mu, I_{m}\right),
$$

where $\mu=V^{T} \Lambda^{-0.5} \hat{\mu}$. We will carry out the computations in the reduced coordinates $\theta$. This reduces the effective dimension of the problem from $N^{2}$ (4096 for our finest grid) to $m=30$. The model reduction follows naturally from assuming that the permeability is smooth, so that the prior is correlated, and the probability mass localizes in parameter space. A similar observation, in connection with data assimilation, was made in [9].

3.2. Multiple grids and adjoint-based BFGS optimization. Implicit sampling requires minimization of $F$ in (3) which in reduced coordinates of this problem takes the form

$$
F(\theta)=\frac{1}{2} \theta^{T} \theta+\frac{1}{2}(z-M P(\theta))^{T} R^{-1}(z-M P(\theta)),
$$

where $M$ is a $k \times N^{2}$ matrix that defines at which locations on the (fine) grid we collect the pressure. We solve the optimization problem using BFGS coupled to an adjoint code to compute the gradient of $F$ with respect to $\theta$ (see also, e.g., [23; 34]).

The adjoint calculations are as follows. The gradient of $F$ with respect to $\theta$ is

$$
\nabla_{\theta} F(\theta)=\theta+\left(\nabla_{\theta} P(\theta)\right)^{T} W,
$$

where $W=-M^{T} R^{-1}(z-M P(\theta))$ and $P$ is an $N^{2}$ vector that contains the pressure on the grid. We use the chain rule to derive $\left(\nabla_{\theta} P(\theta)\right)^{T} W$ as follows:

$$
\begin{aligned}
\left(\nabla_{\theta} P(\theta)\right)^{T} W & =\left(\nabla_{K} P(\theta) \frac{\partial K}{\partial \hat{K}} \frac{\partial \hat{K}}{\partial \theta}\right)^{T} W \\
& =\left(\nabla_{K} P(\theta) e^{\hat{K}} V \Lambda^{0.5}\right)^{T} W=\left(V \Lambda^{0.5}\right)^{T}\left(\nabla_{K} P(\theta) e^{\hat{K}}\right)^{T} W,
\end{aligned}
$$

where $e^{\hat{K}}$ is an $N^{2} \times N^{2}$ diagonal matrix whose elements are the exponentials of the components of $\hat{K}$. The gradient $\nabla_{K} P(\theta)$ can be obtained directly from our finite element discretization. Let $P=P(\theta)$, let $K_{l}$ be the $l$-th component of $K$, and take the derivative with respect to $K_{l}$ of our finite element discretization to obtain

$$
\frac{\partial P}{\partial K_{l}}=-A^{-1} \frac{\partial A}{\partial K_{l}} P
$$


where $A$ is the $N^{2} \times N^{2}$ matrix that defines the linear system we solve and where $\partial A / \partial K_{l}$ are componentwise derivatives. We use this result to obtain

$$
\left(\nabla_{K} P(\theta) e^{\hat{K}}\right)^{T} W=-\left(e^{\hat{K}}\right)^{T}\left[\begin{array}{c}
P^{T} \frac{\partial A}{\partial K_{1}}\left(A^{-T} W\right) \\
\vdots \\
P^{T} \frac{\partial A}{\partial K_{N^{2}}}\left(A^{-T} W\right)
\end{array}\right] .
$$

When $P$ is available, the most expensive part in (16) is to evaluate $A^{-T} W$, which is equivalent to solving the adjoint problem (which is equal to itself for this selfadjoint problem). The rest can be computed elementwise by the definition of $A$. Note that there are only a fixed number of nonzeros in each $\partial A / \partial K_{l}$ so that the additional work for solving the adjoint problem in (16) is about $O\left(N^{2}\right)$, which is small compared to the work required for the adjoint solve.

Collecting terms we finally obtain the gradient

$$
\begin{aligned}
\nabla_{\theta} F(\theta) & =\theta+\left(V \Lambda^{0.5}\right)^{T}\left(\nabla_{K} P(\theta) e^{\hat{K}}\right)^{T} W \\
& =\theta-\left(V \Lambda^{0.5}\right)^{T}\left(e^{\hat{K}}\right)^{T}\left[\begin{array}{c}
P^{T} \frac{\partial A}{\partial K_{1}}\left(A^{-T} W\right) \\
\vdots \\
P^{T} \frac{\partial A}{\partial K_{N^{2}}}\left(A^{-T} W\right)
\end{array}\right] .
\end{aligned}
$$

Multiplying by $\left(V \Lambda^{0.5}\right)^{T}$ to go back to physical coordinates will require additional work of $O\left(m N^{2}\right)$. Note that the adjoint calculations for the gradient require only one adjoint solve because the forward solve (required for $P$ ) has already been done before the gradient calculation in the BFGS algorithm. In summary, our adjoint solves are only slightly more expensive than the forward solves. This concludes our derivation of an adjoint method for gradient computations.

We use this adjoint-based gradient computations in a BFGS method with a cubic interpolation line search [32, Chapter 3]. We use the multiple-grids approach to reduce the number of fine-grid solves. We use three grids, $16 \times 16,32 \times 32$, and $64 \times 64$. The required number of iterations on each grid and the number of forward solves are summarized in Table 1. After converting the cost of coarse/medium-grid solves to the cost of fine-grid solves, we estimate the cost of the multiple-grid

\begin{tabular}{|ccc|}
\hline Grid & Iterations & Forward solves \\
\hline $16 \times 16$ & 9 & 32 \\
$32 \times 32$ & 6 & 14 \\
$64 \times 64$ & 5 & 12 \\
\hline
\end{tabular}

Table 1. Required iterations and function evaluations for multiple-grid optimization. 
optimization with 17 fine-grid solves. Without multiple grids, 36 fine-grid solves are needed to find the same minimum.

3.3. Implementation of the random and linear maps. We generate samples using the linear map and random map methods described above. Both require the Hessian of $F$ at the minimum. A direct finite difference method for the Hessian would require $m(m+1)=930$ forward solves, which is too expensive (infeasible if $m$ becomes larger). For that reason, we approximate the Hessian by

$$
H \approx \hat{H}=I-Q^{T}\left(Q Q^{T}+R\right)^{-1} Q,
$$

where $Q=M \nabla_{\theta} P$, as is standard in LMAP methods [24]. Here the gradient of the pressure (or the Jacobian) is computed with finite differences, which requires $m+1$ forward solves.

With this approximate Hessian, generating samples with the random map method requires solving (6) with the ansatz (7). We use a Newton method for solving these equations and observe that it usually converges quickly (within 1-4 iterations). Each iteration requires a derivative of $F$ with respect to $\lambda$, which we implement using the adjoint method, so that each iteration requires two forward solutions. In summary, the random map method requires between 2-8 forward solutions per sample. The linear map method requires generating a Gaussian sample and weighting it by (10) so that only one forward solve is required per sample.

The quality of the weighted ensembles of the random and linear map methods can be assessed by the variance of the weights. A well distributed ensemble has a small variance of the weights. The variance of the weights is equal to $R-1$, where

$$
R=\frac{E\left(w^{2}\right)}{E(w)^{2}} .
$$

In fact, $R$ itself can be used to measure the quality of the samples [1;41]. If the variance of the weights is small, then $R \approx 1$. Moreover, the effective sample size, i.e., the number of unweighted samples that would be equivalent in terms of statistical accuracy to the set of weighted samples, is about $M / R$ [41], where $M$ is the number of samples we draw. In summary, an $R$ close to 1 indicates a well distributed ensemble.

We compute a value of $R$ of about 1.6 for both methods. In fact, we generate 10 synthetic data sets, run implicit sampling with random and linear maps on each set, and estimate $R$ based on $10^{4}$ samples for each numerical experiment. We compute an $R=1.68 \pm 0.10$ for the linear map method and $R=1.63 \pm 0.066$ for the random map method. The random map method thus performs slightly better; however, the cost per sample is also slightly larger (because generating a sample requires solving (6), which in turn requires solving the forward problem). Since the 
linear map method is less expensive and easier to program, it is a more appropriate technique for this problem.

We have also experimented with symmetrization of implicit sampling [22], which is similar in spirit to the classic trick of antithetic variates [25]. The symmetrization of the linear map method is as follows. Sample $\xi$, and compute a sample $x^{+}=\mu+\xi$. Use the same $\xi$ to compute $x^{-}=\mu-\xi$. Then pick $x^{+}$ with probability $p^{+}=w\left(x^{+}\right) /\left(w\left(x^{+}\right)+w\left(x^{-}\right)\right)$and pick $x^{-}$with probability $p^{-}=w\left(x^{-}\right) /\left(w\left(x^{+}\right)+w\left(x^{-}\right)\right)$, and assign the weight $w^{s}=\left(w\left(x^{+}\right)+w\left(x^{-}\right)\right) / 2$. This symmetrization can lead to a smaller $R$, i.e., a better distributed ensemble, in the small noise limit. In our example, we compute the quality measure $R=1.4$. While this $R$ is smaller than for the nonsymmetrized methods, the symmetrization does not pay off in this example since each sample of the symmetrized method requires two forward solves (to evaluate the weights).

3.4. Comparisons with other methods. The MAP and LMAP methods estimate parameters by computing the MAP point, i.e., the most likely parameters in view of the data, and estimate the uncertainty by a Gaussian whose covariance is the inverse of the Hessian of $F$ at the minimum [7; 24; 34; 35]. In our example, LMAP overestimates the uncertainty since the Gaussian approximation has a standard deviation of 0.93 for the first parameter $\theta_{1}$, whereas we compute 0.64 with the linear map and random map methods. The reason for the over-estimation of the uncertainty with LMAP is that the posterior is not Gaussian. This effect is illustrated in Figure 3, where we show histograms of the marginals of the posterior for the first four parameters $\theta_{1}, \theta_{2}, \theta_{3}$, and $\theta_{4}$ along with their Gaussian approximation as in LMAP. We also compute the skewness and excess kurtosis for these marginal densities. While the marginals for the parameters may become "more Gaussian" for the higher-order coefficients of the KL expansion, the joint posterior exhibits significant non-Gaussian behavior. Since implicit sampling (with random or linear maps) does not require linearizations or Gaussian assumptions, it can correctly capture these non-Gaussian features. In the present example, accounting for the non-Gaussian effects brings about a significant reduction of the uncertainty.

Note that code for LMAP can be converted into an implicit sampling code. In particular, implicit sampling with linear maps requires the MAP point and an approximation of the Hessian at the minimum. Both can be computed with LMAP codes. Non-Gaussian features of the posterior can then be captured by weighted sampling with linear maps, where each sample comes at a cost of a single forward simulation.

Another important class of methods for solving Bayesian parameter estimation problems is MCMC. We compare implicit sampling with Metropolis MCMC [28], where we use an isotropic Gaussian proposal density, for which we tuned the variance to achieve an acceptance rate of about $30 \%$. This method requires one 

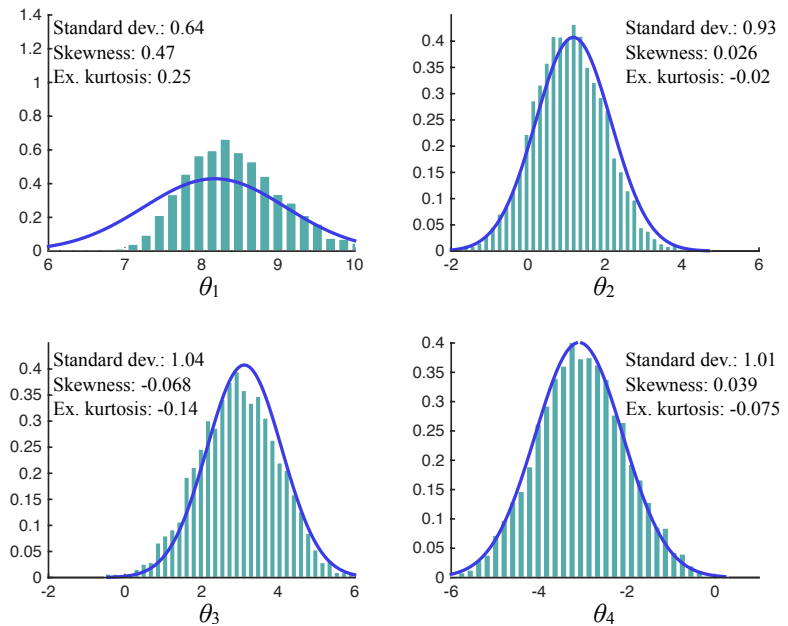

Figure 3. Marginals of the posterior computed with implicit sampling with random maps and their Gaussian approximation obtained via LMAP. Top left: $p\left(\theta_{1} \mid z\right)$. Top right: $p\left(\theta_{2} \mid z\right)$. Bottom left: $p\left(\theta_{3} \mid z\right)$. Bottom right: $p\left(\theta_{4} \mid z\right)$.

forward solution per step (to compute the acceptance probability). We start the chain at the MAP (to reduce burn-in time). In Figure 4, we show the approximation of the conditional mean of the variables $\theta_{1}, \theta_{2}$, and $\theta_{3}$ as a function of the number
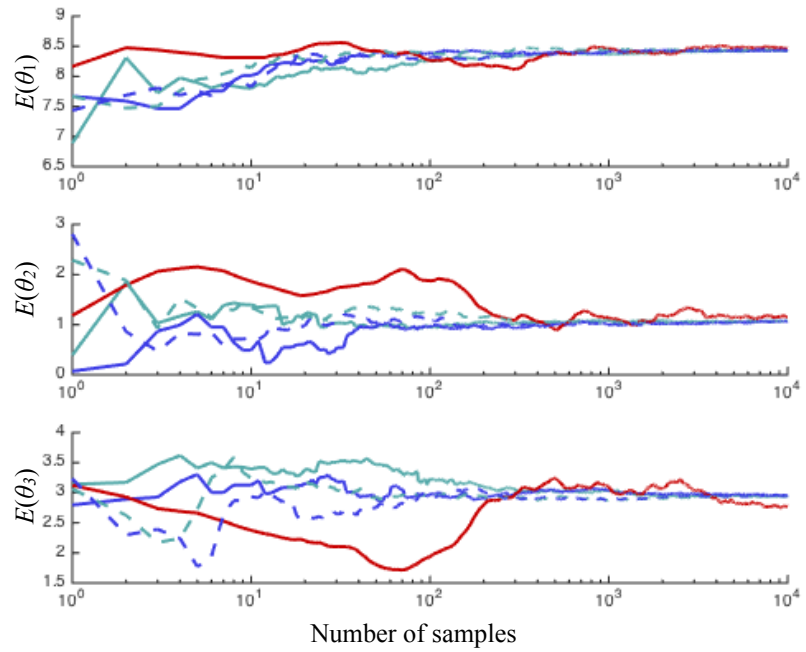

$\begin{array}{lll}- \text { Random map (approx. Hessian) } & \text { Linear map (approx. Hessian) }\end{array}-$ MCMC

Figure 4. Expected value as a function of the number of samples. Red: MCMC. Turquoise: implicit sampling with random maps and approximate Hessian (dashed) and finite difference Hessian (solid). Blue: implicit sampling with linear maps and approximate Hessian (dashed) and finite difference Hessian (solid). 

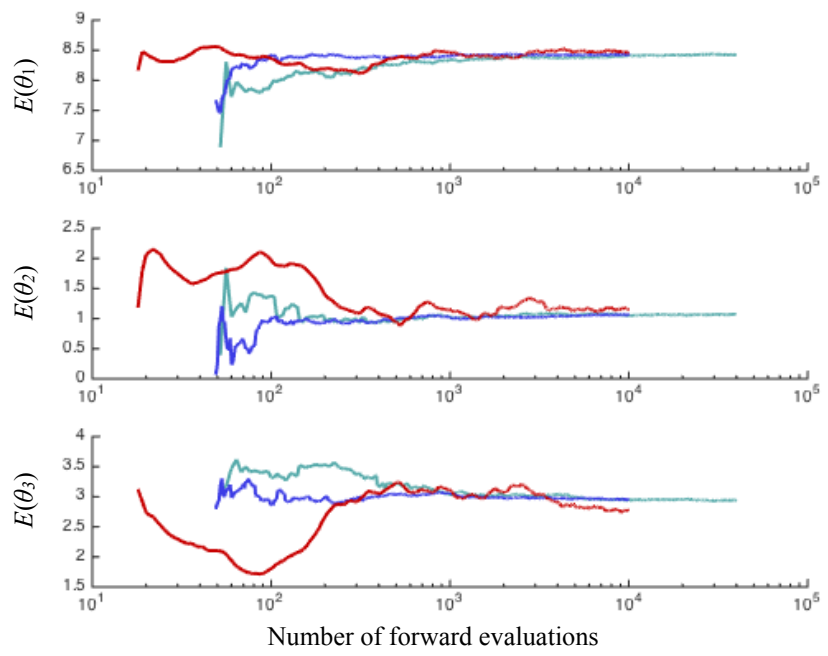

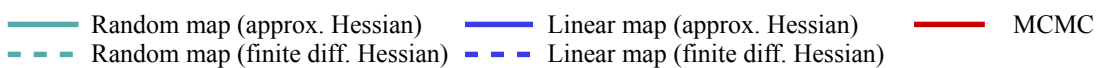

Figure 5. Expected value as a function of required forward solves. Red: MCMC. Turquoise: implicit sampling with random maps and approximate Hessian (dashed) and finite difference Hessian (solid). Blue: implicit sampling with linear maps and approximate Hessian (dashed) and finite difference Hessian (solid).

of steps in the chain. We observe that, even after $10^{4}$ steps, the chain has not settled, in particular for the parameter $\theta_{3}$ (see bottom pane).

With implicit sampling, we observe a faster convergence, in the sense that the approximated conditional mean does not change significantly with the number of samples. In fact, about $10^{2}$ samples are sufficient for accurate estimates of the conditional mean. As a reference solution, we also show results we obtained with implicit sampling (with both random and linear maps) for which we used a Hessian computed with finite differences (rather than with the approximation in (17)).

The cost per sample of implicit sampling and the cost per step of Metropolis MCMC are different, and a fair comparison of these methods should take these costs into account. In particular, the offset cost of the minimization and computation of the Hessian, required for implicit sampling, must be accounted for. We measure the cost of the algorithms by the number of forward solves required. The results are shown for the parameters $\theta_{1}, \theta_{2}$, and $\theta_{3}$ in Figure 5.

We find that the fast convergence of implicit sampling makes up for the relatively large a priori cost (for minimization and Hessian computations). In fact, the figure suggests that the random method requires only a few hundred samples, whereas Metropolis MCMC requires thousands of samples. The convergence of Metropolis MCMC can perhaps be increased by further tuning or by choosing a more advanced transition density. Implicit sampling on the other hand requires little tuning other 
than deciding on standard tolerances for the optimization. Moreover, implicit sampling generates independent samples with a known distribution so that issues such as determining burn-in times, auto-correlation times, and acceptance ratios do not arise. It should also be mentioned that implicit sampling is easy to parallelize. Parallelizing Metropolis MCMC on the other hand is not trivial because it is a sequential technique.

Finally, we discuss connections of our proposed implicit sampling methods to stochastic Newton MCMC [29]. In stochastic Newton, one first finds the MAP point (as in implicit sampling or LMAP) and then starts a number of MCMC chains from the MAP point. The transition probabilities are based on local information about $F$ and make use of the Hessian of $F$, evaluated at the location of the chain. Thus, at each step, a Hessian computation is required which, with our finite difference scheme, requires 31 forward solves (see above) and, therefore, is expensive (compared to generating samples with implicit sampling, which requires computing the Hessian only once). Second-order adjoints (if they were available) do not reduce that cost significantly. We have experimented with stochastic Newton in our example and have used 10-50 chains and taken about 200 steps per chain. Without significant tuning, we find acceptance rates of only a few percent, leading to a slow convergence of the method. We also observe that the Hessian may not be positive definite at all locations of the chain and, therefore, cannot be used for a local Gaussian transition probability. In summary, we find that stochastic Newton MCMC is impractical unless second-order adjoints are available to speed up the Hessian computations. Variations of stochastic Newton were explained and compared to each other in [36].

\section{Conclusions}

We explained how to use implicit sampling to estimate the parameters in PDE from sparse and noisy data. The idea in implicit sampling is to find the most likely state, often called the maximum a posteriori (MAP) point, and generate samples that explore the neighborhood of the MAP point. This strategy can work well if the posterior probability mass localizes around the MAP point, which is often the case when the data constrain the parameters. We discussed how to implement these ideas efficiently in the context of parameter estimation problems using multiple grids and adjoints to speed up the required optimization.

Our implicit sampling approach has the advantage that it generates independent samples so that issues connected with MCMC, e.g., estimation of burn-in times, auto-correlations of the samples, or tuning of acceptance ratios, are avoided. Our approach is also fully nonlinear and captures non-Gaussian features of the posterior 
(unlike linear methods such as the linearization about the MAP point) and is easy to parallelize.

We illustrated the efficiency of our approach in numerical experiments with an elliptic inverse problem that is of importance in applications to reservoir simulation/management and pollution modeling. The elliptic forward model is discretized using finite elements, and the linear equations are solved by balancing domain decomposition by constraints. The optimization required by implicit sampling is done with a BFGS method coupled to an adjoint code. We use the fact that the solutions are expected to be smooth for model order reduction based on KarhunenLoève expansions and found that our implicit sampling approach can exploit this low-dimensional structure. Moreover, implicit sampling is about an order of magnitude faster than Metropolis MCMC sampling (in the example we consider). We also discussed connections and differences of our approach with linear/Gaussian methods, such as linearization about the MAP, and with stochastic Newton MCMC methods. In particular, one can build an implicit sampling code starting from a MAP code by simply adding the Gaussian sampling and weighting step. At the cost of one additional forward solve per sample, the implicit sampling approach can reveal non-Gaussian features.

\section{Acknowledgements}

This material is based upon work supported by the U.S. Department of Energy, Office of Science, Office of Advanced Scientific Computing Research, Applied Mathematics program under contract DE-AC02005CH11231 and by the National Science Foundation under grants DMS-0955078, DMS-1115759, DMS-1217065, and DMS-1419069.

\section{References}

[1] M. S. Arulampalam, S. Maskell, N. Gordon, and T. Clapp, A tutorial on particle filters for online nonlinear/non-Gaussian Bayesian tracking, IEEE T. Signal. Proces. 50 (2002), no. 2, 174-188.

[2] E. Atkins, M. Morzfeld, and A. J. Chorin, Implicit particle methods and their connection with variational data assimilation, Mon. Weather Rev. 141 (2013), no. 6, 1786-1803.

[3] J. Bear and A. Verruijt, Modeling groundwater flow and pollution, Theory and Applications of Transport in Porous Media, no. 2, Reidel, Dordrecht, Holland, 1987.

[4] T. Bengtsson, P. Bickel, and B. Li, Curse-of-dimensionality revisited: collapse of the particle filter in very large scale systems, Probability and statistics: essays in honor of David A. Freedman (D. Nolan and T. Speed, eds.), Inst. Math. Stat. Collect., no. 2, Inst. Math. Stat., Beachwood, OH, 2008, pp. 316-334. MR 2009k:93144 Zbl 1166.93376

[5] P. Bickel, B. Li, and T. Bengtsson, Sharp failure rates for the bootstrap particle filter in high dimensions, Pushing the limits of contemporary statistics: contributions in honor of Jayanta K. Ghosh (B. Clarke and S. Ghosal, eds.), Inst. Math. Stat. Collect., no. 3, Inst. Math. Stat., Beachwood, OH, 2008, pp. 318-329. MR 2010c:93107 
[6] D. Braess, Finite elements: theory, fast solvers, and applications in solid mechanics, Cambridge University, 1997. MR 98f:65002 Zbl 0894.65054

[7] T. Bui-Thanh, O. Ghattas, J. Martin, and G. Stadler, A computational framework for infinitedimensional Bayesian inverse problems, I: The linearized case, with application to global seismic inversion, SIAM J. Sci. Comput. 35 (2013), no. 6, A2494-A2523. MR 3126997 Zbl 1287.35087

[8] A. J. Chorin and O. H. Hald, Stochastic tools in mathematics and science, 3rd ed., Texts in Applied Mathematics, no. 58, Springer, New York, 2013. MR 3076304 Zbl 06150329

[9] A. J. Chorin and M. Morzfeld, Conditions for successful data assimilation, J. Geophys. Res. Atmos. 118 (2003), no. 20, 11522-11533.

[10] A. J. Chorin, M. Morzfeld, and X. Tu, Implicit particle filters for data assimilation, Commun. Appl. Math. Comput. Sci. 5 (2010), no. 2, 221-240. MR 2011m:60118 Zbl 1229.60047

[11] _ Implicit sampling, with application to data assimilation, Chin. Ann. Math. Ser. B 34 (2013), no. 1, 89-98. MR 3011460 Zbl 1261.62084

[12] A. J. Chorin and X. Tu, Implicit sampling for particle filters, P. Natl. Acad. Sci. USA 106 (2009), no. $41,17249-17254$.

[13] M. Dashti and A. M. Stuart, Uncertainty quantification and weak approximation of an elliptic inverse problem, SIAM J. Numer. Anal. 49 (2011), no. 6, 2524-2542. MR 2873245 Zbl 1234.35309

[14] C. R. Dohrmann, A preconditioner for substructuring based on constrained energy minimization, SIAM J. Sci. Comput. 25 (2003), no. 1, 246-258. MR 2004k:74099 Zbl 1038.65039

[15] A. Doucet, S. Godsill, and C. Andrieu, On sequential Monte Carlo sampling methods for Bayesian filtering, Stat. Comput. 10 (2000), no. 3, 197-208.

[16] Y. Efendiev, T. Hou, and W. Luo, Preconditioning Markov chain Monte Carlo simulations using coarse-scale models, SIAM J. Sci. Comput. 28 (2006), no. 2, 776-803. MR 2007b:65009 Zbl 1111.65003

[17] T. A. El Moselhy and Y. M. Marzouk, Bayesian inference with optimal maps, J. Comput. Phys. 231 (2012), no. 23, 7815-7850. MR 2972870 Zbl 06117578

[18] R. P. Fedorenko, A relaxation method for solving elliptic difference equations, USSR Comp. Math. Math. 1 (1962), no. 4, 1092-1096. Zbl 0163.39303

[19] R. G. Ghanem and P. D. Spanos, Stochastic finite elements: a spectral approach, Springer, New York, 1991. MR 91k:73102 Zbl 0722.73080

[20] J. Goodman, K. K. Lin, and M. Morzfeld, Small-noise analysis and symmetrization of implicit Monte Carlo samplers, Commun. Pur. Appl. Math. (2015), online publication July.

[21] J. Goodman and A. D. Sokal, Multigrid Monte Carlo method: conceptual foundations, Phys. Rev. D 40 (1989), no. 6, 2035-2071.

[22] J. Goodman and J. Weare, Ensemble samplers with affine invariance, Commun. Appl. Math. Comput. Sci. 5 (2010), no. 1, 65-80. MR 2011d:65007 Zbl 1189.65014

[23] M. Hinze, R. Pinnau, M. Ulbrich, and S. Ulbrich, Optimization with PDE constraints, Mathematical Modelling: Theory and Applications, no. 23, Springer, New York, 2009. MR 2010h:49002 Zbl 1167.49001

[24] M. A. Iglesias, K. J. H. Law, and A. M. Stuart, Evaluation of Gaussian approximations for data assimilation in reservoir models, Comput. Geosci. 17 (2013), no. 5, 851-885. MR 3104638 
[25] M. H. Kalos and P. A. Whitlock, Monte Carlo methods, vol. 1, Wiley, New York, 1986. MR 88e:65009 Zbl 0655.65004

[26] O. P. Le Maître and O. M. Knio, Spectral methods for uncertainty quantification: with applications to computational fluid dynamics, Springer, New York, 2010. MR 2011b:65002 Zbl 1193.76003

[27] J. S. Liu and R. Chen, Blind deconvolution via sequential imputations, J. Am. Stat. Assoc. 90 (1995), no. 430, 567-576. Zbl 0826.62062

[28] J. S. Liu, Monte Carlo strategies in scientific computing, Springer, New York, 2008. MR 2010b: 65013 Zbl 1132.65003

[29] J. Martin, L. C. Wilcox, C. Burstedde, and O. Ghattas, A stochastic Newton MCMC method for large-scale statistical inverse problems with application to seismic inversion, SIAM J. Sci. Comput. 34 (2012), no. 3, A1460-A1487. MR 2970260 Zbl 1250.65011

[30] M. Morzfeld and A. J. Chorin, Implicit particle filtering for models with partial noise, and an application to geomagnetic data assimilation, Nonlinear Proc. Geophys. 19 (2012), 365-382.

[31] M. Morzfeld, X. Tu, E. Atkins, and A. J. Chorin, A random map implementation of implicit filters, J. Comput. Phys. 231 (2012), no. 4, 2049-2066. MR 2012m:62287 Zbl 1242.65012

[32] J. Nocedal and S. J. Wright, Numerical optimization, 2nd ed., Springer, New York, 2006. MR 2007a:90001 Zbl 1104.65059

[33] D. S. Oliver, Minimization for conditional simulation: relationship to optimal transport, J. Comput. Phys. 265 (2014), 1-15. MR 3173132

[34] D. S. Oliver and Y. Chen, Recent progress on reservoir history matching: a review, Computat. Geosci. 15 (2011), no. 1, 185-221. Zbl 1209.86001

[35] D. S. Oliver, A. C. Reynolds, and N. Liu, Inverse theory for petroleum reservoir characterization and history matching, Cambridge University, 2008.

[36] N. Petra, J. Martin, G. Stadler, and O. Ghattas, A computational framework for infinitedimensional Bayesian inverse problems, II: Stochastic Newton MCMC with application to ice sheet flow inverse problems, SIAM J. Sci. Comput. 36 (2014), no. 4, A1525-A1555. MR 3233941 Zbl 1303.35110

[37] C. E. Rasmussen and C. K. I. Williams, Gaussian processes for machine learning, MIT, Cambridge, MA, 2006. MR 2010i:68131 Zbl 1177.68165

[38] N. Recca, A new methodology for importance sampling, master's thesis, New York University, 2011.

[39] C. Snyder, T. Bengtsson, P. Bickel, and J. Anderson, Obstacles to high-dimensional particle filtering, Mon. Weather Rev. 136 (2008), no. 12, 4629-4640.

[40] A. M. Stuart, Inverse problems: a Bayesian perspective, Acta Numer. 19 (2010), 451-559. MR 2011i:65093 Zbl 1242.65142

[41] E. Vanden-Eijnden and J. Weare, Data assimilation in the low noise regime with application to the Kuroshio, Mon. Weather Rev. 141 (2013), no. 6, 1822-1841.

[42] B. Weir, R. N. Miller, and Y. H. Spitz, Implicit estimation of ecological model parameters, Bull. Math. Biol. 75 (2013), no. 2, 223-257. MR 3022352 Zbl 1310.92005

[43] V. S. Zaritskii, V. B. Svetnik, and L. I. Shimelevich, Monte-Carlo technique in problems of optimal information processing, Automat. Remote Control 36 (1975), no. 12, 2015-2022. MR 55 \#1701 Zbl 0344.60041 
Received June 23, 2015.

MATTHiAs MORZFELD: mmo@math.lbl.gov

Department of Mathematics, University of California, Berkeley, Evans Hall, Berkeley, CA 94720, United States

and

Lawrence Berkeley National Laboratory, 1 Cyclotron Road, Berkeley, CA 94720, United States

Xuemin Tu: xtu@math.ku.edu

Department of Mathematics, University of Kansas, 1460 Jayhawk Boulevard, Lawrence, KS 66045, United States

JON WILKENING: wilken@math.berkeley.edu

Department of Mathematics, University of California, Berkeley, Evans Hall, Berkeley, CA 94720, United States

and

Lawrence Berkeley National Laboratory, 1 Cyclotron Road, Berkeley, CA 94720, United States

AlEXANDRE J. ChORIN: chorin@math.1bl.gov

Department of Mathematics, University of California, Berkeley, Evans Hall, Berkeley, CA 94720, United States

and

Lawrence Berkeley National Laboratory, 1 Cyclotron Road, Berkeley, CA 94720, United States 


\title{
Communications in Applied Mathematics and Computational Science
}

\author{
msp.org/camcos
}

EDITORS

MANAGING EDITOR

John B. Bell

Lawrence Berkeley National Laboratory, USA

jbbell@lbl.gov

\section{BOARD OF EDITORS}

\begin{tabular}{|c|c|c|c|}
\hline Marsha Berger & $\begin{array}{l}\text { New York University } \\
\text { berger@cs.nyu.edu }\end{array}$ & Ahmed Ghoniem & $\begin{array}{l}\text { Massachusetts Inst. of Technology, USA } \\
\text { ghoniem@mit.edu }\end{array}$ \\
\hline Alexandre Chorin & $\begin{array}{l}\text { University of California, Berkeley, USA } \\
\text { chorin@math.berkeley.edu }\end{array}$ & Raz Kupferman & $\begin{array}{l}\text { The Hebrew University, Israel } \\
\text { raz@math.huji.ac.il }\end{array}$ \\
\hline Phil Colella & $\begin{array}{l}\text { Lawrence Berkeley Nat. Lab., USA } \\
\text { pcolella@lbl.gov }\end{array}$ & Randall J. LeVeque & $\begin{array}{l}\text { University of Washington, USA } \\
\text { rj1@ amath.washington.edu }\end{array}$ \\
\hline Peter Constantin & $\begin{array}{l}\text { University of Chicago, USA } \\
\text { const@cs.uchicago.edu }\end{array}$ & Mitchell Luskin & $\begin{array}{l}\text { University of Minnesota, USA } \\
\text { luskin@umn.edu }\end{array}$ \\
\hline Maksymilian Dryja & $\begin{array}{l}\text { Warsaw University, Poland } \\
\text { maksymilian.dryja@acn.waw.pl }\end{array}$ & Yvon Maday & $\begin{array}{l}\text { Université Pierre et Marie Curie, France } \\
\text { maday@ann.jussieu.fr }\end{array}$ \\
\hline M. Gregory Forest & $\begin{array}{l}\text { University of North Carolina, USA } \\
\text { forest@amath.unc.edu }\end{array}$ & James Sethian & $\begin{array}{l}\text { University of California, Berkeley, USA } \\
\text { sethian@ math.berkeley.edu }\end{array}$ \\
\hline Leslie Greengard & $\begin{array}{l}\text { New York University, USA } \\
\text { greengard@ cims.nyu.edu }\end{array}$ & Juan Luis Vázquez & $\begin{array}{l}\text { Universidad Autónoma de Madrid, Spain } \\
\text { juanluis.vazquez@uam.es }\end{array}$ \\
\hline Rupert Klein & $\begin{array}{l}\text { Freie Universität Berlin, Germany } \\
\text { rupert.klein@pik-potsdam.de }\end{array}$ & Alfio Quarteroni & $\begin{array}{l}\text { Ecole Polytech. Féd. Lausanne, Switzerland } \\
\text { alfio.quarteroni@epfl.ch }\end{array}$ \\
\hline \multirow[t]{2}{*}{ Nigel Goldenfeld } & $\begin{array}{l}\text { University of Illinois, USA } \\
\text { nigel@uiuc.edu }\end{array}$ & Eitan Tadmor & $\begin{array}{l}\text { University of Maryland, USA } \\
\text { etadmor@cscamm.umd.edu }\end{array}$ \\
\hline & & Denis Talay & $\begin{array}{l}\text { INRIA, France } \\
\text { denis.talay@inria.fr }\end{array}$ \\
\hline
\end{tabular}

\section{PRODUCTION}

production@msp.org

Silvio Levy, Scientific Editor

See inside back cover or msp.org/camcos for submission instructions.

The subscription price for 2015 is US $\$ 85 /$ year for the electronic version, and $\$ 120 /$ year $(+\$ 15$, if shipping outside the US) for print and electronic. Subscriptions, requests for back issues from the last three years and changes of subscribers address should be sent to MSP.

Communications in Applied Mathematics and Computational Science (ISSN 2157-5452 electronic, 1559-3940 printed) at Mathematical Sciences Publishers, 798 Evans Hall \#3840, c/o University of California, Berkeley, CA 94720-3840, is published continuously online. Periodical rate postage paid at Berkeley, CA 94704, and additional mailing offices.

CAMCoS peer review and production are managed by EditFLOW ${ }^{\circledR}$ from MSP.

\section{PUBLISHED BY}

mathematical sciences publishers

nonprofit scientific publishing

http://msp.org/

(C) 2015 Mathematical Sciences Publishers 


\section{Communications in Applied Mathematics and Computational Science}

vol. 10

no. 2

2015

A Nitsche-based cut finite element method for a fluid-structure interaction problem

André Massing, Mats G. Larson, Anders Logg and Marie E.

ROGNES

An adaptive multiblock high-order finite-volume method for solving the shallow-water equations on the sphere

Peter McCorquodale, Paul A. Ullrich, Hans Johansen and Phillip Colella

Low Mach number fluctuating hydrodynamics of binary liquid mixtures

Andy Nonaka, Yifei Sun, John B. Bell and Aleksandar Donev

Parameter estimation by implicit sampling

Matthias Morzfeld, Xuemin Tu, Jon Wilkening and

AleXAndRe J. Chorin 